\title{
Support Systems Designed for Older Drivers to Achieve Safe and Comfortable Driving
}

\author{
Anna Bjelkemyr ${ }^{1}$, Tania Dukic ${ }^{2}$, Rachel Owens ${ }^{3}$, Torbjorn Falkmer ${ }^{3,45,6}$, Hoe C. Lee ${ }^{3 *}$ \\ ${ }^{1}$ SAFER Vehicle and Traffic Safety Centre at Chalmers, Gothenburg, Sweden \\ ${ }^{2}$ Swedish National Road and Transport Research Institute (VTI), Gothenburg, Sweden \\ ${ }^{3}$ School of Occupational Therapy \& Social Work, CHIRI, Curtin University, Perth, Australia \\ ${ }^{4}$ Department of Rehabilitation, School of Health Sciences, Jönköping University, Jönköping, Sweden \\ ${ }^{5}$ Rehabilitation Medicine, Department of Medicine and Health Sciences (IMH), Faculty of Health Sciences, \\ Linköping University \& Pain and Rehabilitation Centre, Linköping, Sweden \\ ${ }^{6}$ School of Occupational Therapy, La Trobe University, Melbourne, Australia \\ Email: *Hoe.Lee@curtin.edu.au
}

Received March 5, 2013; revised April 11, 2013; accepted May 9, 2013

Copyright (C) 2013 Anna Bjelkemyr et al. This is an open access article distributed under the Creative Commons Attribution License, which permits unrestricted use, distribution, and reproduction in any medium, provided the original work is properly cited.

\begin{abstract}
Background: The number of older people is increasing. Many of them expect to maintain a rich social life and to continue driving at an older age. Objective: The present study investigates the mechanisms behind self-regulation and driving cessation in order to suggest development of support systems to prolong older drivers' safe mobility. Method: Three focus groups were conducted with 19 older active drivers aged 65+ who were divided according to annual mileage driven. Results: A content analysis revealed broad self-regulatory behaviour as already reported in the literature, e.g., avoiding driving at rush hour and at night. The participants also reported difficulty in finding the way to their final destination and an increasing need to plan their travelling. Co-piloting was a behaviour applied by couples to cope with difficulties encountered in traffic. A large part of the discussion was focused on emerging feelings of stress, anxiety and fear when driving in recent years, a feeling induced by external factors e.g., other road users' behaviour, traffic density or high speed. Apart from health problems, high levels of stress could explain driving cessation, especially for women. An increased feeling of safety and comfort could be achieved by an increased use of support systems specifically designed to respond to older drivers' needs. Conclusion: Support systems for older drivers should increase comfort and decrease their stress levels. New systems, such as co-pilot function and more developed Global Positioning System (GPS) supporting of the entire travel from door to door, should be developed to respond to the market needs.
\end{abstract}

Keywords: Older Driver; Safe Mobility; Self-Regulation; Driver Cessation; Support System

\section{Introduction}

The population is ageing resulting in a changing demography. In Sweden it is predicted that the population aged $65+$ will increase to about $30 \%$ of the total population by 2030 [1]. Not only are older persons increasing in number, but also they are becoming healthier and richer, and they expect to maintain a high quality of life. It is therefore important to find the best way to treat age-related complaints or illnesses, as well as to plan how society will adjust to meet the future needs and demands of an older population wishing to maintain their mobility.

Driving for shopping and leisure increases after retirement before decreasing again at an older age. Individual driving habits are likely to be maintained to an

\footnotetext{
*Corresponding author.
}

older age [2]. Maintaining one's mobility and independence has been shown to prevent social isolation, and so may help with preserving one's good health, given the association between social isolation and depression [3,4]. The mobility that comes with using a car can increase self-confidence, prestige and self-esteem [5], and driving and owning a car is associated with youth and status [6]. However, becoming older can imply physical, sensory and/or cognitive limitations, which have consequences on driving [7-9] e.g., lower body mobility; impaired vision and hearing; slower ability to react; dementia; Parkinson's disease and other psychological and cognitive diagnosis affecting driving behavior.

Older drivers want to maintain a safe and comfortable mobility [10]. For most old people, age-related physical, sensory and cognitive limitations, which may affect their 
driving, evolve over a long period of time. During this period of continuously decreasing health, it is likely that there would be an increasing amount of self-regulation, in order to maintain a safe mobility so as to continue with practical and social everyday activities. Self-regulation can manifest as avoiding certain conditions (e.g. driving at night or during rush hour) or difficult traffic situations (e.g. driving through specific intersections) [11,12]. Further, this self-regulating behaviour can lead to absolute driving cessation [13]. Many older drivers are over-anxious and less confident about their driving ability and may make the decision to cease driving earlier than necessary [11]. On the other hand, there is evidence that not all people are able to self-regulate to compensate for the negative effects of age and it seems that this is partly associated with gender [12].

The present study investigates the mechanisms behind self-regulation and driving cessation, in order to suggest the development of support systems that may prolong older drivers' safe mobility. In particular, the study looks at whether in-vehicle supporting systems help maintain a comfortable and safe mobility. Another area of focus is on gender and mileage (i.e., annual distance driven) since these two factors seem to be of importance for older drivers' safe mobility [14]. This paper presents results from three focus groups characterised by different mileages, and focuses on the participants' opinions about their needs and limitations as drivers, as well as their awareness of their self-regulation and thoughts about driving cessation.

\section{Methods}

\subsection{Participants}

The focus group study took place in Sweden in the city of Jönköping. The city, which has a population of 84,423 inhabitants, is Sweden's $9^{\text {th }}$ largest city with an area of $44.33 \mathrm{~km}^{2}$ and is equipped with a public transport system. It was important for the study that there was an alternative to driving to allow for individual preferences in the choice of means of transport. It was also important that different traffic environments were represented in a close range to the city (e.g. city centre driving, main road driving and motorway driving) so that it was possible to choose different routes and still go from the same point A to $B$.

\subsection{Participants}

A total of 19 participants were recruited through a pensioner organization in Jönköping municipality. The criteria to take part in the study included being older than 65 years, being retired, having a driving license and being an active driver. The age of 65 is the legislated age of retirement in Sweden and in this article it defines when a person is considered to be old. The participants were divided into three groups based on their annual mileage driven in the last year: high-mileage ( $>12,000 \mathrm{~km} /$ year), medium-mileage (7000 - 12,000 km/year) and low- mileage ( $<7000 \mathrm{~km} /$ year) (see Table 1). It is worth noting that dividing the participants into annual mileage groups resulted in an uneven gender distribution. The low- mileage group included only women and the high-mileage group included only men, while the medium-mileage group was mixed. All participants reported that they lived an active life with family and friends, and that they participated in leisure activities. They all lived in their own household, either alone or with their partner. The health of the participants varied but no participant was in need of regular geriatric care. Two women in the lowmileage group did not have access to a car in their household. All participants were asked to report if they drove a large amount, a medium amount or a small amount at this point of life. The male drivers who estimated that they drove a large amount drove around 25,000 km/year while the women who estimated they drove a large amount drove around 10,000 km/year. For the male and female participants who responded that they drove a small amount the annual distance driven were 6000 and 1000 $\mathrm{km} /$ year respectively.

\subsection{Focus Group}

Focus groups were used to collect the data [15]. This methodology is common within the field of qualitative research and was important in the present study to gain indepth knowledge regarding older drivers' support needs to continue driving in the future. The interviews of the three focus groups were performed in the same manner and by the same moderator. The moderator was assisted by a researcher for technical support.

Four main themes were discussed during the interview: 1) mobility patterns, 2) self-regulation, 3 ) driving cessation, and 4) vehicle support systems. The interview was organized in two parts. The first part aimed to cover the participants' mobility pattern, their perceived driving selfregulation in the last year and the question of when and why they would stop driving in the future.

After a short break, the second part started by showing

Table 1. Characteristics of the participants included in the three mileage groups (female/male).

\begin{tabular}{ccccc}
\hline Mileage & Number & $\begin{array}{c}\text { Mean } \\
\text { Age }\end{array}$ & $\begin{array}{c}\text { Mean Driving } \\
\text { License Age }\end{array}$ & $\begin{array}{c}\text { Mean Distance } \\
\text { Driven Last } \\
\text { Year (km) }\end{array}$ \\
\hline Low & $6 /-$ & $72 /-$ & $29 /-$ & $2100 /-$ \\
Medium & $4 / 5$ & $73 / 75$ & $27 / 19$ & $7200 / 8700$ \\
High & $0 / 4$ & $-/ 77$ & $-/ 22$ & $-/ 15,500$ \\
\hline
\end{tabular}


three short video-clips of city, main road and highway driving to anchor the interview regarding infrastructure and driving behaviour in real traffic situations. The intention of this part of the interview was to investigate their perception of limitations as well as their use of support systems. The interviews lasted three hours each, including the break. They were recorded using a digital voice recorder and all recordings were transcribed. A content analysis was performed on the transcribed material [16]. The analyses were made with data analysis software MAXQDA [17]. The data were categorized into themes and subthemes. The themes were identified to be the same as the four main focus group themes. The subthemes are the subjects that the participants talked about when asking them questions about mobility pattern, self-regulation, driving cessation and support systems. A total of 34 subthemes were identified and divided into six "travel needs" (necessary travels, social/pleasure, family, cottage, sport/group activities and nature), eight "individual circumstances" (life quality, time, age, gender, living conditions, health, relations and economy), nine "outer circumstances" (road design, wild animals, society infrastructure, parking, other road users, time of year, darkness, weather conditions and traffic density), five "means of transport" (walking, bicycling, car, public transport and aviation/train/touring coach), three "traffic environment” (city traffic, main road and highway), "stress/worry/anxiety" and "environmental influence". A subgroup "irrelevant" was added to assure that all data were analysed. The occurrence of each instance belonging to a subtheme was counted to quantitatively describe the proportions of the different subthemes in the material. The discussions during the focus group interviews were rich and broad and only the sub-themes that represented at least $10 \%$ of the discussions are presented in the result section. To illustrate participants' views, some quotes are included in the text. These were translated from Swedish to English.

\section{Results}

The distribution of sub-themes per theme is summarised in Table 2. The percentage represents the part of the discussion devoted to each sub-theme by theme.

\subsection{Mobility Patterns}

Musselwhite's classification was used to analyse the mobility pattern [18]. The authors classified travelling into three categories: travelling to fulfil practical needs, e.g., shopping; travelling to fulfil social needs, e.g., visiting family and friends; and travelling for aesthetic needs, e.g., to see the ocean.

Overall, the consistent result from all the participants was that practical and social travelling was performed by
Table 2. Main themes and sub-themes discussed in the three focus groups.

\begin{tabular}{ccc}
\hline Theme & Subtheme & $\%$ \\
\hline Mobility pattern & $\begin{array}{c}\text { Travel types (practical, social and } \\
\text { aesthetic needs) }\end{array}$ & 52 \\
Gender & 14 \\
Living & 14 \\
Time of year & 10 \\
Self-regulation & Quality of life & 10 \\
& Self-regulation actions & 64 \\
Driving cessation & Stress & 36 \\
& Health & 48 \\
& Stress & 33 \\
\hline
\end{tabular}

means of a car. Trips for practical reasons were reported on a daily or weekly basis and a majority of the participants in all three groups mentioned that they travelled by car once a week to a superstore outside the city centre. The shopping bought on this weekly trip was heavy and so public transport was not considered to be an alternative. A majority of the participants also reported travelling to visit friends and family members, to participate in activities for pensioners and to go to sport activities. Visits to family often included travelling a long distance since family members were spread over the country. Additionally, more than half of the participants had summer cottages, which they travelled to on a regular basis. These participants claimed that as long as they had their summer cottage they would also need their car. In most cases public transport was not an alternative for these trips, due to poor accessibility.

Participants in the medium- and high-mileage groups reported travelling to fulfil aesthetic needs. In particular, they enjoyed driving to the coast or the forest and being able to make spontaneous decisions to go somewhere other than where they had initially planned to go. One participant said, "Sometimes it gets a bit dull at home and so we go out for a drive and a look around". The majority of these trips are performed during summer time.

Although the car was the preferred means of transport some exceptions were found. The first exception mentioned was going on a vacation trip or weekend trip. Here planes, train and coach were the preferred means of transport. The participants in the high-mileage group agreed that this resulted in a lack of freedom but mentioned that the advantages outweighed the disadvantages. The second exception counted for two women in the low- 
mileage group who described an alternative way of living and reported that public transport was their main means of transport. They lived without a car in a one person household in the city centre near all facilities and drove only on rare occasions. They described a life where attending some activities was difficult and some places were impossible to reach. One of them said, "You just have to get used to your situation and not go to some places."

The third exception was related to gender. When the household had only one car, female participants reported that the women were more likely to choose an alternative transport, either going by public transport or bicycle, or walking.

Lastly, a difference between men and women was reported when the couple was travelling together in the car.

The men were often the ones who drove, while the women tended to be passengers.

\subsection{Self-Regulation}

Participants in all groups reported self-regulating while driving to avoid physical or mental discomfort. Throughout the discussions, an increased feeling of discomfort and numerous changes in driving behaviour due to age were reported. Examples of experienced self-regulatory actions were (from the most common): reducing speed, avoiding motorways, avoiding big cities, avoiding long distance travels, avoiding unknown cities, avoiding driving in darkness, avoiding overtaking, avoiding poor weather, avoiding being a hindrance, spending more time planning journeys and avoiding rush hours. One important reason for several of these self-regulations was due to being retired, which, in turn, led to being more flexible and not having to travel at a specific time or on a specific date. This type of self-regulation was considered to be positive. All groups reported to be calmer, less reckless and less daring drivers than earlier in life. Additionally, participants in the low-mileage group drove mostly in the immediate area or on familiar roads.

Different types of self-regulation actions were reported in different traffic environments. In city traffic, all three groups reported that the volume of traffic had increased over the years and other drivers were less forgiving. The high- and medium-mileage groups talked about road signs. They described their difficulty in orienting themselves and finding their destination in unknown cities due to numerous signs. One man said, "Signs are popping up everywhere these days (laughs)". The low-mileage group reported an increased need to thoroughly plan their driving in cities. This was due to a combination of not knowing which route to take given the increased traffic density and a less forgiving traffic culture.

In regards to self-regulation on main-roads, there were problems with cars coming up from behind and several participants talked about letting them overtake at suitable parking areas or small road entries along the main road. An experience that increased with age was the sensation that cars could appear from anywhere and this was accompanied by difficulties in estimating the speed of vehicles approaching from the side when entering a main road. One solution was to slow down or stop before entering the main road. One man said, "I prefer to stop when a car is approaching from further up the road as I can't tell if they are going at 50 or $90 \mathrm{~km} / \mathrm{h}$ ".

When discussing motorway driving the participants described the situation as very stressful due to the high speed and the disrespect from other road users, particularly when overtaking or being overtaken, and entering and exiting the motorway. One woman said, "You always have to be alert when you're on the motorway".

In all three groups a large part of the self-regulation discussion was devoted to how participants were more stressed, worried, anxious and scared when driving in recent years. The three groups did not use the same words but, within each group, participants were consistent with the chosen word throughout the discussion. These feelings were mentioned as a reason for avoiding some traffic situations and choosing alternative roads.

In the high-mileage group they described themselves as being stressed and the discussion covered how to avoid this feeling. One man said, "If you're stressed on the journey, you're quite a different person when you arrive". In the medium-mileage group the feeling of worry was discussed. This increased worry was common in many situations in life but was also applicable for driving. In the low-mileage group the participants described feeling scared. One woman referred to a discussion she had had with a female friend lately: "Some drivers get scared, I believe women mostly. It happens when you get older, even if you have been driving a lot. Suddenly, it just happens". Another woman claimed that being 'insecure' would be a more correct description of how she felt. Participants believed that being scared in traffic happened more often to women than to men.

Self-regulating behaviour was reported to be a consequence of physical and cognitive age-related limitations that they felt affected their driving. Poor vision and difficulty in rotating their head, neck and body were limitations perceived in all three groups. At intersections, this led to a visually "dead angle". Poor vision was discussed several times. In the low-mileage group two of the women had more severe visual problems due to cataracts and macular degeneration. The woman with a cataract diagnosis described how this influenced her driving: "I drove to the summer cottage. It was a little dark and I drove at $50 \mathrm{~km} / \mathrm{h}$ although the speed limit was $70 \mathrm{~km} / \mathrm{h}$. Other cars came up from behind me and beeped at me and I tried to stop to let them pass. It was so annoying. I 
would never have behaved like that before. I would have just gone faster!”

Further, part of the self-regulatory behaviour was different for men and women when travelling together with their partner. They reported how specific situations were handled as a team, i.e., the passenger as co-pilot. When the driver was the man, the woman supported the driver with information such as whether the road from the side was free from traffic at intersections. Several of the female participants described how they drove to the outskirt of bigger or unknown cities where they changed driver and their male partner drove the last part of the journey.

\subsection{Driving Cessation}

Participants were asked about the future, such as whether they planned to cease driving and for what reasons. One main reason was their health condition. Visual problems due to age were well understood and considered to be a strong factor in being a safe or an unsafe driver. The participants were aware of the influence of a medical diagnosis on when to make the decision to discontinue driving. Participants claimed that they expected to realise by themselves when their health conditions were too bad to continue driving. However, they also expected their close family, such as their partner, as well as their children and grandchildren, to stop them if they were no longer fit to drive. The process of thinking of ceasing to drive depended on the mileage group. In the high-mileage group they had thought about it but still considered their immediate future to include driving. The medium-mileage group described how they believe that ceasing to drive would include a successive phasing out of driving. They had faith that this phase would come naturally and that they would be well aware when they were no longer safe drivers. In the low-mileage group, the participants hoped to have some awareness and to understand when it was time for them to stop driving. However, they considered their partners to have less insight into their own driving ability and so worried about how to talk to them about not driving anymore. It was considered a sensitive subject and participants thought the best way to handle this was by letting a medical expert inform their partner that they should not drive anymore.

An increased feeling of stress was also a reason to cease driving. The low-mileage group described being frightened in specific traffic situations and as a conesquence not daring to drive anymore. These problems were believed to be more common for older female drivers. One woman was aware of this being the case for herself, reporting that she was getting older and had started to feel more insecure in traffic: "I should start thinking about not driving anymore... perhaps, in the near future". The discussion of when to stop driving lead into a conversation about the importance of practicing their driving in case their partner became unable to drive anymore or passed away.

To stop driving was not always a decision that the participants made directly. It could be a consequence of the participants having moved to the city centre since they could not take care of their houses anymore and felt more comfortable living in the city. One man described how he and his wife had moved closer to the city centre to be prepared for a time when he would have less strength and perhaps not be able to drive anymore.

\subsection{Support System}

The participants drove cars from different manufacturers and production years ranging from 1995 to 2010 (Median = 2003). Depending on what car they drove, their experiences of vehicle support systems differed, though support systems were reported to be used by all participants. Most of them were used to compensate for physiccal limitations and increase driver comfort. One system reported was electronic rear mirrors. These mirrors help with parking by automatically tilting downwards so that the driver can see the painted lines of the parking space. Backing sensors were also considered helpful. Participants perceived an increased difficulty in parking the car without assistance from these sensors due to an increased stiffness in their head, neck and body. Having automatic transmission was likewise highly valued. A man described how he became a safer driver, "There is only one thing you need to think about and that is using the brakes. You don't need to think about changing gear”. From a female participant, "it was so tough! I was getting pain in my hip, especially when I had to drive in the city centre”. Cruise control and adjustable seats were thought to increase comfort while driving particularly when having problems with mobility in the legs and hips. Navigation systems were also highly appreciated. One woman said, "It is really good to drive with a navigator. It says "turn here and turn there." You know exactly how to get to where you're going."

\section{Discussion}

The aim of this study was to investigate the mechanisms behind self-regulation and driving cessation in order to suggest the development of support systems to prolong older drivers' safe mobility. Overall, all participants reported travelling in their own car to fulfil practical and social needs. All groups reported travelling by car to do the weekly shopping and visit the health care centre, family and friends, and to participate in activities for pensioners, or in sports, as well as going to cultural activities. Travelling by car for leisure was still common for the high- and medium-mileage groups but not for the 
low-mileage group. Older people who had moved closer to the city centre in preparation for a time when they would not be able to drive anymore found that their need for a car had decreased.

Gender could explain many differences in the mobility pattern of the older drivers. In the low-mileage group, there were only female participants, whereas only male participants were present in the high-mileage group. Women not only drove shorter distances, but their driving histories were different from that of the male participants: male participants received their driving license an average eight years earlier than the female participants. Additionally, the subjective evaluation of driving a lot or just a little did not mean the same distance for men and women when putting them into different mileage groups. These findings indicate a difference in attitude towards driving and towards themselves as drivers that is both socially and culturally set at an early age, and that is maintained at an older age. Today, there is still a small gender difference with $52.5 \%$ of men and $47.5 \%$ of women having a driving licence in 2012 compared to $60 \%$ and $40 \%$ respectively in 1980 [19].

The change in mobility patterns with age is accompanied by self-regulatory behaviour. Results showed that self-regulation when driving was just one of several life adaptations to ageing. Participants in both the high- and medium-mileage groups believed that generations born in the forties and later were well aware of the process of getting older and adapted their way of living accordingly. This covered selling the house and summer cottage, and moving to a smaller apartment strategically located near the city centre, public transport and health care centre. Self-regulatory behaviour related to driving was reported by all participants and was considered to be a positive experience. Reported self-regulation, e.g., avoiding driving at night or in poor weather conditions, is consistent with previous literature $[11,13,20,21]$. Self-regulation in the low- and medium-mileage groups included planning journeys more thoroughly, avoiding large and unknown cities and avoiding being a hindrance. This indicates that these two groups have progressed further in the continuous process of self-regulation. They are closer to the decision to discontinue driving permanently than the participants in the high-mileage group.

An accurate judgment of when to cease driving is difficult to make for medical experts, friends and family, and most of all for the driver. The main discussion in all groups covered the discomfort of feeling stressed/worried/scared when driving. This phenomenon has been reported earlier by Persson [22] who found that $20 \%$ of participants cited feeling nervous as the main reason to stop driving. The feeling of discomfort plays an important role when choosing between taking your own car or another means of transport. An overall impression from the present study is that feeling comfortable is the highest priority when driving. Security is likely a part of feeling comfortable, however, comfort, both physical and mental, is the keyword. Gender and age are predictors of stress level where older drivers are more likely to experience stress than younger drivers, and women are more likely to be stressed than men [23]. This might result in older people, and women in particular, being overanxious and less confident about their ability. This could result in a decision to cease driving too early [11,23-25]. This is a two-way problem. Drivers with poor self-esteem stop driving too early whereas drivers with high self-esteem continue to drive for too long. By decreasing the feeling of discomfort, stress, worry, anxiety, nervousness or being scared many older drivers can probably continue to drive safely and comfortably for a longer time period. Women are three times more likely to stop driving than men [26]. Stress reported by participants could come from a wide range of sources e.g., other road users such as truck drivers following closely before overtaking, infrastructure design such as high speed roads with alternating $2+1$ lane system or large cities where it is difficult to find your destination. Several countermeasures could be used and developed to increase safe mobility for older drivers. Stress coming from other road users is difficult to prevent since it is more related to attitudes and behaviour towards older drivers. Stress coming from the infrastructure or different manoeuvres could be solved with help of support systems.

In general, support systems for older drivers should increase their comfort and decrease their stress levels. Systems that compensate for physical and psychological reductions of capacity are available on the market and may already be used by older drivers. Participants in the present study spoke highly of electric rear mirrors that facilitate parking and of cars with automatic transmission. Cars with automatic transmission have been shown to decrease the number of required tasks while driving [27]. Furthermore, there are systems available that have not been advertised or marketed at older drivers, e.g., navigation systems. Navigation systems may be useful in managing the stress associated with high-speed roads and becoming lost, as well as the increased need to plan car journeys. In 2007, Monash University proposed developing a "safer mode" in the navigation system. This system could be used to take drivers on a route that avoided black spots e.g., difficult intersections. The system could be further developed to include information on how to avoid high speed roads, road works, roads with a high traffic flow, as well as information on available parking spots close to the final destination. Such technology is already available and needs to be adapted to fit older drivers' needs. Further research and development is needed to investigate the more specific needs of older 
drivers in road situations. Challenges for the future are to develop new support systems to tackle older drivers' specific difficulties at intersections.

Another type of support system to assist older drivers is the co-pilot aid [28]. In the future, the co-piloting function could be replaced by a technical system that supports good decisions from the planning through to the driving stage. The design should take into account both relevant safety aspects and also those aspects related to comfort and decreasing stress. This is a challenge that the automotive industry will have to face in the near future.

The recruitment of participants was made via pensioner organizations representing $41.5 \%$ [29] of the target population. However, focus groups in qualitative studies do not necessarily have to be representative as their strengths are shown in the variability and richness of the groups' answers. Another limitation may have been the unequal distribution of gender across groups. Women were overrepresented in the low-mileage group while the number of men was overrepresented in the high-mileage group. However, as the proportion of women among high-mileage older drivers is very low in reality it was judged acceptable to compose the groups accordingly.

In conclusion, stress, fear and anxiety are feelings that were difficult to define but seem to become an increaseingly important part of driving with age. Furthermore, they are expressed differently by men and women. Important research for the future would be to understand whether these feelings are relevant in indicating the level of self-regulation needed, as well as choosing a time to stop driving. Research should also investigate whether this perceived stress can be successfully suppressed with a support system, such as planning aid or a co-pilot system. In particular, it is recommended that future research should look at systems for travel planning before getting into the car and instantaneous in-car travel planning (copilot system). Finally, more research about safety support systems that address the problems of "looking around" and awareness of other road users is needed.

\section{Acknowledgements}

We acknowledge SAFER, Vehicle and Traffic Safety Centre at Chalmers, Gothenburg, Sweden, for funding this research and the participants from the pensioner organisations PRO and SPF in Jönköping, Sweden.

\section{REFERENCES}

[1] SCB, Statistics Sweden, “Trends and Forecasts 2011 Population, Education and Labour Market in Sweden- Outlook to Year 2030," Statistics Sweden, Forecast Institute, 2012.

http://www.scb.se/statistik/_publikationer/UF0515_2012

\section{A01_BR_AM85BR1201.pdf}

[2] R. J. Hjorthol, L. Levin and A. Sirén, "Mobility in DifFerent Generations of Older Persons. The Development of Daily Travel in Different Cohorts in Denmark, Norway and Sweden,” Journal of Transport Geography, Vol. 18, No. 5, 2010, pp. 624-633. http://dx.doi.org/10.1016/j.jtrangeo.2010.03.011

[3] R. A. Marottoli, C. F. Mendes de Leon, T. A. Glass, C. S. Williams, L. M. Cooney Jr. and L. F. Berkman, "Consequences of Driving Cessation: Decreased out of Home Activity Levels," Journal of Gerontology: Series B: Psychological Science \& Social Science, Vol. 55, No. 6, 2000, pp. 334-340.

[4] S. J. Fonda, R. B. Wallace and A. R. Herzog, “Changes in Driving Patterns and Worsening Depressive Symptoms among Older Adults,” Journal of Gerontology: Social Sciences, Vol. 56, No. 6, 2001, pp. 343-351.

[5] A. Ellaway, S. Mactyrine, R. Hiscock and A. Kearns, "In the Driving Seat: Psychosocial Benefits from Private Motor Vehicle Transport Compared to Public Transport," Transport Research Part F, Vol. 6, No. 3, 2003, pp. 217231. http://dx.doi.org/10.1016/S1369-8478(03)00027-5

[6] A. Siren and L. Hakamies-Blomqvist, "Sense and Sensibility: A Narrative Study of Older Women's Car Driving,” Transport Research Part F, Vol. 8, No. 3, 2005, pp. 213-228. http://dx.doi.org/10.1016/j.trf.2005.04.008

[7] S. Classen, A. Horgas, K. Awadzi, B. Messinger-Rapport, O. Shechtman and Y. Joo, "Clinical Predictors of Older Driver Performance on a Standardized Road Test," Traffic Injury Prevention, Vol. 9, No. 5, 2008, pp. 456-462. http://dx.doi.org/10.1080/15389580802260026

[8] R. Fuller, "Towards a General Theory of Driver Behaviour,” Accident Analysis and Prevention, Vol. 37, No. 3, 2005, pp. 461-472.

http://dx.doi.org/10.1016/j.aap.2004.11.003

[9] H. C. Lee, A. H Lee, D. Cameron and C. Li-Tsang, "Using a Driver Simulator to Identify Older Drivers Inflated Risk of Motor Vehicle Crashes," Journal of Safety Research, Vol. 34, No. 4, 2003, pp. 453-459. http://dx.doi.org/10.1016/j.jsr.2003.09.007

[10] H. Summala, “Towards Understanding Motivational and Emotional Factors in Driver Behaviour: Comfort through Satisficing,” In: P. C. Cacciabue, Ed., Modelling Driver Behaviour in Automotive Environments: Critical Issues in Driver Interactions with Intelligent Transport Systems, Springer, London, 2007, pp. 189-207.

[11] M. R. J. Baldock, J. L. Mathias, A. J. McLean and A. Berndt, "Self-Regulation of Driving and Its Relationship to Driving Ability among Older Adults," Accident Analysis and Prevention, Vol. 38, No. 5, 2006, pp. 1038-1045. http://dx.doi.org/10.1016/j.aap.2006.04.016

[12] J. Charlton, J. Oxley, B. Fildes, P. Oxley, S. Newstead, S. Koppel and M. O’Hare, "Characteristics of Older Drivers Who Adopt Self-Regulatory Driving Behaviours,” Transport Research Part F, Vol. 9, No. 5, 2006, pp. 363-373.

[13] L. Hakamies-Blomqvist and B. Wahlström, "Why Do Older Drivers Give Up Driving?” Accident Analysis and Prevention, Vol. 30, No. 3, 1998, pp. 305-312. http://dx.doi.org/10.1016/S0001-4575(97)00106-1

[14] B. N. Fildes, "Future Directions for Older Research,” Traf- 
fic Injury Prevention, Vol. 9, No. 4, 2008, pp. 387-393. http://dx.doi.org/10.1080/15389580802272435

[15] R. Kreuger, "Focus Groups: A Practical Guide for Applied Research,” 2nd Edition, Sage, California, 1994.

[16] U. H. Graneheim and B. Lundman, "Qualitative Content Analysis in Nursing Research: Concepts, Procedures and Measure to Achieve Trustworthiness," Nurse Education Today, Vol. 24, No. 2, 2004, pp. 105-112. http://dx.doi.org/10.1016/j.nedt.2003.10.001

[17] MAXQDA, “Distribution by VERBI GmbH,” 1995. http://www.maxqda.com

[18] C. Musselwhite and H. Haddad, "Prolonging the Safe Driving of Older People through Technology,” University of West England, Final Report, 2007.

[19] Transportstyrelsen, “Statistics on Driving Licence,” 2012. http://www.transportstyrelsen.se/sv/Press/Statistik/Vag/K orkort/Statistik-over-korkortsinnehavare-efter-kon/

[20] J. Eberhard, “Safe Mobility of Senior Citizens,” Journal of the International Association of Traffic Safety Sciences, Vol. 20, No. 1, 1996, pp. 29-37.

[21] L. J. Molnar and D. W. Eby, "The Relationship between Self-Regulation and Driving-Related Abilities in older Drivers: An Exploratory Study," Traffic Injury Prevention, Vol. 9, No. 4, 2008, pp. 314-319. http://dx.doi.org/10.1080/15389580801895319

[22] D. Persson, “The Elderly Driver: Deciding When to Stop,” The Gerontologist, Vol. 33, No. 1, 1993, pp. 88-91. http://dx.doi.org/10.1093/geront/33.1.88

[23] J. D. Hill and L. Ng Boyle, "Driver Stress as Influenced by Driver Maneuvers and Roadway Conditions,” Transport Research Part F, Vol. 10, No. 3, 2007, pp. 177-186.

[24] J. Oxley, J. Charlton, J. Scully and S. Koppel, “Older Female Drivers: An Emerging Transport Safety and Mobility Issue in Australia," Accident Analysis and Prevention, Vol. 42, No. 2, 2010, pp. 515-522. http://dx.doi.org/10.1016/j.aap.2009.09.017

[25] A. Siren and L. Hakamies-Blomqvist, "Does Gendered Driving Create Gendered Mobility? Community-Related Mobility in Finnish Women and Men Aged 65+," Transport Research Part F, Vol. 9, No. 5, 2006, pp. 374-382.

[26] C. A. Unsworth, Y. Wells, C. Browning, S. A. Thomas and H. Kendig, "To Continue, Modify or Relinguish Driving: Findings from a Longitudinal Study of Healthy Ageing," Gerontology, Vol. 53, No. 6, 2007, pp. 423-431. http://dx.doi.org/10.1159/000111489

[27] H. Selander, I. Bolin and T. Falkmer, "Does Automatic Transmission Improve Driver Behaviour in Older Drivers?” Gerontology, Vol. 58, No. 2, 2012, pp. 181-187. http://dx.doi.org/10.1159/000329769

[28] B. H. Vrkljan and M. J. Polgar, "Driving, Navigation, and Vehicular Technology: Experiences of Older Drivers and Their Co-Pilots,” Traffic Injury Prevention, Vol. 8, No. 4, 2007, pp. 403-410. http://dx.doi.org/10.1080/15389580701576423

[29] SCB, Statistics Sweden, "Associational Life in Sweden: General Welfare Production, Social Capital, Training in Democracy,” 2003.

http://www.scb.se/statistik/LE/LE0101/2000I02/LE98SA 0301.pdf 